\title{
AN INTRODUCTION TO MULTIVARIATE KRAWTCHOUK POLYNOMIALS AND THEIR APPLICATIONS
}

\author{
Persi Diaconis*and Robert Griffiths ${ }^{\dagger}$ \\ Stanford University and Oxford University
}

Version 1.2

October 29, 2018

\begin{abstract}
Orthogonal polynomials for the multinomial distribution $m(\boldsymbol{x}, \boldsymbol{p})$ of $N$ balls dropped into $d$ boxes (box $i$ has probability $p_{i}$ ) are called multivariate Krawtchouk polynomials. This paper gives an introduction to their properties, collections of natural Markov chains which they explicitly diagonalize and associated bivariate multinomial distributions.
\end{abstract}

\section{Introduction}

This paper relates orthogonal polynomials, Markov chains and statistical modeling. Bill Studden loved all of these topics and their interactions. The setting is a $d$-category multinomial distribution of $N$ balls dropped into $d$ boxes with probabilities $\boldsymbol{p}=\left(p_{1}, \ldots, p_{d}\right)$. Throughout $p_{i}>0$ and the multinomial distribution is

$$
m(\boldsymbol{x}, \boldsymbol{p})=\left(\begin{array}{c}
N \\
x_{1}, \cdots, x_{d}
\end{array}\right) \prod_{i=1}^{d} p_{i}^{x_{i}}, \quad 0 \leq x_{i}, \sum_{i=1}^{d} x_{i}=N
$$

Systems of orthogonal polynomials for the multinomial are defined in Griffiths (1971). They generalize Krawtchouk polynomials for the Binomial and are

${ }^{*}$ Department of Statistics, Sequoia Hall, 390 Serra Mall, Stanford University, Stanford, California 94305-4065, USA.

${ }^{\dagger}$ Department of Statistics, University of Oxford, 1 South Parks Rd, Oxford, OX1 3TG, UK; email griff@stats.ox.ac.uk 
called multivariate Krawtchouk polynomials. A self contained development of these along with properties and pointers to an emerging literature is in section two.

There is not a unique set of orthogonal polynomials in higher dimensions than one because the ordering of powers $x_{1}^{n_{1}} \cdots x_{d}^{n_{d}}$ is not unique within a fixed degree $|n|=n_{1}+\cdots+n_{d}$. The definitions here lead to constructing a unique set of polynomials by choosing an orthonormal basis of functions on $\{1,2, \ldots, d\}$, see the definitions in section 2.1. Further orthogonal polynomials in a particular set are uniquely defined by their multiple leading coefficients (lemma 1 in section 2.1). This allows us to show that the polynomials suggested by Grunbaum and Rahman (2011) and Xu (2013) fit into our definition.

Multivariate Krawtchouk polynomials appear as the eigen-functions of a variety of natural Markov chains generalizing the classical Ehrenfest urn. Consider an urn containing $N$ balls in $d$ colours. A ball is chosen at random and its colour changed to $j$ with probability $p_{j}$. This can be generalized in various directions. The balls can be partitioned into groups in a general way. Balls in the $\alpha^{\text {th }}$ group have their colours changed in a general way: a ball of colour $i$ is changed to colour $j$ with probability $P_{\alpha}(i, j)$. With appropriate choices, all of these chains are diagonalized by multivariate Krawtchouk polynomials. This work captures chains previously studied by Hoare and Rahman (2008), Khare and Zhou (2009), Zhou and Lange (2009) and Mizukawa (2010), Mizukawa (2011). Multivariate Krawtchouk polynomials also have a universal quality, diagonalizing symmetrized products of general Markov chains. These topics are explained in section three.

The third central topic is bivariate distributions with multinomial margins. This topic has a long history going back to work of Lancaster (1969). Bivariate Lancaster distributions with multinomial margins have an expansion

$$
P(\boldsymbol{x}, \boldsymbol{y})=m(\boldsymbol{x}, \boldsymbol{p}) m(\boldsymbol{y}, \boldsymbol{p})\left\{1+\sum_{n} \rho_{\boldsymbol{n}} h_{\boldsymbol{n}} Q_{\boldsymbol{n}}(\boldsymbol{x}) Q_{\boldsymbol{n}}(\boldsymbol{y})\right\},
$$

with $Q_{n}$ the multivariate Krawtchouk polynomials, and $h_{n}$ given by

$$
\mathbb{E}\left[Q_{\boldsymbol{n}}(\boldsymbol{X}) Q_{\boldsymbol{n}}(\boldsymbol{Y})\right]=\delta_{\boldsymbol{m} \boldsymbol{n}} h_{\boldsymbol{n}} \rho_{\boldsymbol{n}}
$$

The $\rho_{\boldsymbol{n}}$ are called generalized correlations. A basic problem, the Lancaster problem, is what values of $\rho_{\boldsymbol{n}}$ are admissible to have $P(\boldsymbol{x}, \boldsymbol{y}) \geq 0$ ?. This problem was solved in the Binomial case by Eagleson (1969). A useful necessary and sufficient condition appears in section three. This leans on the multinomial hypergroup property which may be of independent interest. 
There are natural choices of $\rho_{\boldsymbol{n}}$; if $K(\boldsymbol{x}, \boldsymbol{y})$ is a reversible Markov chain with Krawtchouk polynomial eigenfunctions, then

$$
P(\boldsymbol{x}, \boldsymbol{y})=m(\boldsymbol{x}, \boldsymbol{p}) K(\boldsymbol{x}, \boldsymbol{y})
$$

has a Lancaster expansion with $\rho_{\boldsymbol{n}}$ the eigen-values of $K$. This paper is a $d$-dimensional version of Diaconis and Griffiths (2012) which works out the connections between generalized Ehrenfest urns with two colours, Lancaster expansions and one variable Krawtchouk polynomials. The theory is more complete (and simpler) in this case and the reader might find it useful motivation.

As usual, new developments raise new questions; what are the extreme points of the $d \times d$ stochastic matrices with $P$ as stationary distribution? Find a full solution of the Lancaster problem. What is the connection to Schur-Weyl duality and Bosonic Fock space? We are sorry not to be able to call on Bill Studden's expert help.

\subsection{Definitions and Background}

The multinomial distribution associated with dropping $N$ balls into $d$ boxes having probabilities $\boldsymbol{p}=\left(p_{1}, \ldots, p_{d}\right)$ is

$$
m(\boldsymbol{x}, \boldsymbol{p})=\left(\begin{array}{c}
N \\
x_{1}, \cdots, x_{d}
\end{array}\right) \prod_{j=1}^{d} p_{j}^{x_{j}}, \quad 0 \leq x_{j}, \sum_{j=1}^{d} x_{j}=N .
$$

Griffiths (1971) defines orthogonal polynomials for $m(\boldsymbol{x}, \boldsymbol{p})$ by choosing a complete set of orthogonal functions $\left\{u_{j}^{(l)}\right\}$ with respect to $\boldsymbol{p}$, for $l=$ $0,1, \ldots, d-1, j=1,2, \ldots, d$. Insist throughout that $u_{j}^{(0)} \equiv 1$. Thus, for all $k, l=0,1, \ldots, d-1$,

$$
\sum_{j=1}^{d} u_{j}^{(k)} u_{j}^{(l)} p_{j}=\delta_{k l} a_{k}
$$

In this paper we usually take these functions to be orthonormal when $a_{k}=1$, $k=1, \ldots, d-1$, unless indicated. Examples of natural choices of $\left\{u^{(l)}\right\}$ are given in the following section 2.2. Often $\left\{u^{(l)}\right\}$ is a basis of eigen-functions for a Markov chain on $[d]=\{1,2, \ldots, d\}$. Writing $\boldsymbol{u}$ for $\left\{u^{(l)}\right\}$, Griffiths (1971) defines a collection of orthogonal polynomials

$$
\left\{Q_{\boldsymbol{n}}(\boldsymbol{x}, \boldsymbol{u})\right\} \text { with } \boldsymbol{n}=\left(n_{1}, \ldots, n_{d-1}\right),|\boldsymbol{n}| \leq N
$$


as the coefficient of $w_{1}^{n_{1}} \cdots w_{d-1}^{n_{d-1}}$ in the generating function

$$
G(\boldsymbol{x}, \boldsymbol{w}, \boldsymbol{u})=\prod_{j=1}^{d}\left\{1+\sum_{l=1}^{d-1} w_{l} u_{j}^{(l)}\right\}^{x_{j}}
$$

For integer $x_{j}$, expanding each term in the product by the binomial expansion gives a polynomial in $x_{j}$ so the coefficient of $\boldsymbol{w}^{\boldsymbol{n}}$ is a polynomial in $\boldsymbol{x}$. It is easy to see that $Q_{\boldsymbol{n}}$ is a polynomial of degree $|\boldsymbol{n}|=n_{1}+\cdots+n_{d-1}$. For example:

$$
\begin{aligned}
\boldsymbol{n} & =(0, \ldots, 0) & & Q_{\boldsymbol{n}} \equiv 1 \\
\boldsymbol{n} & =\left(0, \ldots, 1_{l}, \ldots, 0\right) & & Q_{\boldsymbol{n}}=\sum_{j=1}^{d} u_{j}^{(l)} x_{j} \stackrel{\text { Def }}{=} S_{l} \\
\boldsymbol{n} & =\left(0, \ldots, 1_{l}, \ldots, 1_{m}, \ldots, 0\right) & Q_{\boldsymbol{n}} & =\frac{1}{2} S_{l} S_{m}-\frac{1}{2} \sum_{i=1}^{d} x_{i} u_{i}^{(l)} u_{i}^{(m)} \\
\boldsymbol{n} & =\left(0, \ldots, 2_{l}, \ldots, 0\right) & & Q_{\boldsymbol{n}}=\frac{1}{2} S_{l}^{2}-\frac{1}{2} \sum_{i=1}^{d} x_{i}\left(u_{i}^{(l)}\right)^{2} .
\end{aligned}
$$

It is straightforward to show, using (4), that the $\left\{Q_{\boldsymbol{n}}\right\}$ are orthogonal:

$$
\mathbb{E}\left[Q_{\boldsymbol{n}}(\boldsymbol{X}, \boldsymbol{u}) Q_{m}(\boldsymbol{X}, \boldsymbol{u})\right]=\delta_{\boldsymbol{m n}}\left(\begin{array}{c}
N \\
|\boldsymbol{n}|
\end{array}\right)\left(\begin{array}{c}
|\boldsymbol{n}| \\
\boldsymbol{n}
\end{array}\right) \prod_{j=1}^{d-1} a_{j}^{n_{j}}
$$

In (6), $\boldsymbol{X}$ has a multinomial $m(\boldsymbol{x}, \boldsymbol{p})$ distribution. Griffiths (1971) also gives a related construction of multivariate orthogonal polynomials on the negative multinomial distribution via a generating function approach.

The $\left\{Q_{n}\right\}$ also have an easily verified stability property: their definition does not depend on $N$, as long as $|\boldsymbol{n}| \leq N$ the same $\left\{Q_{\boldsymbol{n}}\right\}$ work for all sufficiently large $N$.

As defined, it is not so clear how to express $Q_{n}$ as a polynomial. Recent work of Mizukawa and Tanaka (2004) and Grunbaum and Rahman (2011) clarifies this. They use hypergeometric notation. Let

$$
F_{1}^{(n)}(-\boldsymbol{m},-\boldsymbol{x} ;-N ; \boldsymbol{u}):=\sum_{k . . \leq N} \frac{\prod_{i=1}^{n}\left(-m_{i}\right)_{\left(k_{i \cdot}\right)} \prod_{j=1}^{n}\left(-x_{j}\right)_{\left(k_{\cdot j}\right)}}{\prod_{i j} k_{i j} !(-N)_{(k .)}} \prod_{i, j} u_{i j}^{k_{i j}}
$$

where a $\cdot$ in an index means sum, eg $k_{\cdot j}=\sum_{i=1}^{d-1} k_{i j}$,

$$
a_{(n)}=a(a+1) \cdots(a+n-1), \quad a_{[n]}=a(a-1) \cdots(a-n+1)
$$

and the sum is over all $n \times n$ matrices $\left(k_{i j}\right)$ with non-negative integer entries with sum of entries at most $N$. 
Proposition 1. Let $\left\{v_{j}^{(l)}\right\}$ be an orthonormal basis, $0 \leq l \leq d-1,1 \leq j \leq d$, with $v_{d}^{(l)} \equiv 1$. For all $l$, let $u_{i j}=1-v_{j}^{(i)}, i, j \in[d-1]$, then with $Q_{m}(\boldsymbol{x}, \boldsymbol{v})$ defined in (3),(41)

$$
Q_{\boldsymbol{m}}(\boldsymbol{x}, \boldsymbol{v})=\frac{N !}{\prod_{i=1}^{d} m_{i} !} F_{1}^{(d-1)}(-\boldsymbol{m},-\boldsymbol{x} ;-N ; \boldsymbol{u}) .
$$

Proof Reduce the variables to $x_{1}, \ldots, x_{d-1}$ by letting $x_{d}=N-\sum_{i=1}^{d-1} x_{i}$, then

$$
\begin{aligned}
G(x, w) & =\prod_{j=1}^{d}\left(1+\sum_{i=1}^{d-1} w_{i} v_{j}^{(i)}\right)^{x_{j}} \\
& =\prod_{j=1}^{d-1}\left(1+\sum_{i=1}^{d-1} w_{i} v_{j}^{(i)}\right)^{x_{j}} \times(1+w .)^{N-\sum_{j=1}^{d-1} x_{j}} \\
& =\prod_{j=1}^{d-1}\left(1+w \cdot-\sum_{i=1}^{d-1} w_{i} u_{i j}\right)^{x_{j}} \times(1+w .)^{N-\sum_{j=1}^{d-1} x_{j}} \\
& =\prod_{j=1}^{d-1}\left(1-\sum_{i=1}^{d-1} \frac{w_{i}}{1+w} u_{i j}\right)^{x_{j}} \times(1+w .)^{N} \\
& =\sum_{k_{i j}}(1+w .)^{N-k .} \prod_{i=1}^{d-1} w_{i}^{k_{i} \cdot} \prod_{i j}\left(-u_{i j}\right)^{k_{i j}} \prod_{j=1}^{d-1} \frac{x_{j}\left[k_{j}\right]}{\prod_{i=1}^{d-1} k_{i j} !} .
\end{aligned}
$$

The coefficient of $\prod_{i=1}^{d-1} w_{i}^{m_{i}}$ in (8) is

$$
\begin{aligned}
& \sum_{k . . \leq N} \frac{\left(N-k_{. .}\right) !}{\prod_{i=1}^{d-1}\left(m_{i}-k_{i .}\right) !} \prod_{i j}\left(-u_{i j}\right)^{k_{i j}} \prod_{j=1}^{d-1} \frac{x_{j\left[k_{j}\right]}}{\prod_{i=1}^{d-1} k_{i j} !} \\
= & \frac{N !}{\prod_{i=1}^{d-1} m_{i} !} \sum_{k . . \leq N} \frac{\prod_{i=1}^{d-1}\left(-m_{i}\right)_{\left(k_{i \cdot}\right)} \prod_{j=1}^{d-1}\left(-x_{j}\right)_{\left(k_{. j}\right)}}{\prod_{i j} k_{i j} !(-N)_{(k . .)}} \prod_{i, j} u_{i j}^{k_{i j}} .
\end{aligned}
$$

Equating coefficients now gives the result.

A well known and easy to check conditional product binomial construction is

$$
m(\boldsymbol{x} ; \boldsymbol{p})=\prod_{j=1}^{d-1} b\left(x_{j}, p_{j} /\left(1-\left|\boldsymbol{p}_{j-1}\right|\right), N-\left|\boldsymbol{x}_{j-1}\right|\right),
$$


where $b(x, p, N)=\left(\begin{array}{c}N \\ x\end{array}\right) p^{x}(1-p)^{N-x}, \boldsymbol{x}_{k}=\left(x_{1}, \ldots x_{k}\right),\left|\boldsymbol{x}_{k}\right|=x_{1}+\cdots+x_{k}$ with similar notation for the $\boldsymbol{p}_{k}$ terms. The way that a set of multidimensional orthogonal polynomials is constructed using (8) is to use 1dimensional Krawtchouk orthogonal polynomials on the conditional distributions. $\mathrm{Xu}(2013)$ gives a unified treatment of multivariate Hahn, Jacobi and Krawtchouk polynomials which are constructed from product conditional distributions. In his treatment there are natural constructions beginning with multivariate Jacobi polynomials which lead to Hahn polynomials via a multinomial-Dirichlet mixture and then from multivariate Hahn polynomials to multivariate Krawtchouk polynomials as a limit when the index parameters in the multinomial-Dirichlet tend to infinity with the ratios tending to $\boldsymbol{p}$. We show in the next theorem that his multivariate Krawtchouk polynomials are a special case of the multivariate Krawtchouk polynomials in this paper. A general preliminary lemma is needed.

Lemma 1. Let $\left\{Q_{\boldsymbol{n}}(\boldsymbol{x})\right\}$ be a d-dimensional orthogonal polynomials set on a random variable $\boldsymbol{X}$. Then the polynomials are uniquely determined by their leading coefficients.

Proof. Take the orthogonal polynomial set to be orthonormal without loss of generality. Denote the reproducing kernel polynomials by

$$
Q_{n}(\boldsymbol{x}, \boldsymbol{y})=\sum_{|\boldsymbol{n}|=n} Q_{\boldsymbol{n}}(\boldsymbol{x}) Q_{\boldsymbol{n}}(\boldsymbol{y}), n=0,1, \ldots
$$

The reproducing kernel orthogonal polynomials are invariant under all choices of multidimensional orthogonal polynomials on the given distribution. Let the leading coefficient of $Q_{\boldsymbol{n}}(\boldsymbol{x})$ be $S_{\boldsymbol{n}}(\boldsymbol{x})$. Then for $|\boldsymbol{n}|=\left|\boldsymbol{n}^{\prime}\right|$

$$
\mathbb{E}\left[S_{\boldsymbol{n}}(\boldsymbol{Y}) Q_{\boldsymbol{n}^{\prime}}(\boldsymbol{Y})\right]=\mathbb{E}\left[Q_{\boldsymbol{n}}(\boldsymbol{Y}) Q_{\boldsymbol{n}^{\prime}}(\boldsymbol{Y})\right]=\delta_{\boldsymbol{n n}^{\prime}},
$$

because $Q_{\boldsymbol{n}^{\prime}}(\boldsymbol{Y})$ is orthogonal to polynomials in $\boldsymbol{Y}$ of degree less than $\left|\boldsymbol{n}^{\prime}\right|$. Thus

$$
\mathbb{E}\left[S_{\boldsymbol{n}}(\boldsymbol{Y}) Q_{n}(\boldsymbol{x}, \boldsymbol{Y})\right]=Q_{\boldsymbol{n}}(\boldsymbol{x}),
$$

uniquely determining $Q_{\boldsymbol{n}}(\boldsymbol{x})$ by $S_{\boldsymbol{n}}(\boldsymbol{x})$ among all orthogonal polynomial sets on $\boldsymbol{X}$.

Because of Lemma 1 to check that two orthogonal polynomials sets on the same distribution are identical (up to normalizing constants) it is sufficient to check that the leading coefficients are proportional. We now calculate the leading coefficients in $\mathrm{Xu}$ (2013)'s orthogonal polynomials on the 
multinomial and show an identity with our multivariate Krawtchouk polynomials. The notation in $\mathrm{Xu}(2013)$ is adapted to agree with notation in this paper. In our orthogonal polynomials $Q_{\boldsymbol{n}}(\boldsymbol{x}, \boldsymbol{u})$ has a single leading term proportional to

$$
S_{\boldsymbol{n}}(\boldsymbol{x})=\prod_{j=1}^{d-1} S_{j}^{n_{j}}, \quad \text { where } \quad S_{j}=\sum_{k=1}^{d} u_{k}^{(j)} x_{k}
$$

with $\left\{u^{(j)}\right\}$ a set of orthogonal functions on $\boldsymbol{p}=\left(p_{1}, \ldots, p_{d}\right)$.

We now describe the conditional binomial constructed polynomials in $\mathrm{Xu}$ (2013). The 1-dimensional Krawtchouk polynomials there are defined by

$$
K_{n}(x ; p, N)={ }_{2} F_{1}\left(-n,-x ;-N ; p^{-1}\right), n=0, \ldots, N,
$$

where, with a standard definition,

$$
{ }_{2} F_{1}(a, b ; c ; z)=\sum_{r=0}^{\infty} \frac{a_{(k)} b_{(k)}}{c_{(k)}} \frac{z^{k}}{k !} .
$$

The scaling is such that $K_{n}(0 ; p, N)=1$. The conditional binomial multidimensional Krawtchouk polynomials are defined by

$$
\begin{aligned}
K_{\boldsymbol{n}}(\boldsymbol{x} ; \boldsymbol{p}, N)=\frac{(-1)^{|\boldsymbol{n}|}}{(-N)_{(|\boldsymbol{n}|)}} \prod_{j=1}^{d-1} \frac{p_{j}^{n_{j}}}{\left(1-\left|\boldsymbol{p}_{j-1}\right|\right)^{n_{j}}}\left(-N+\left|\boldsymbol{x}_{j-1}\right|+\left|\boldsymbol{n}^{j+1}\right|\right)_{\left(n_{j}\right)} \\
\quad \times K_{n_{j}}\left(x_{j} ; \frac{p_{j}}{1-\left|\boldsymbol{p}_{j-1}\right|}, N-\left|\boldsymbol{x}_{j-1}\right|-\left|\boldsymbol{n}^{j+1}\right|\right)
\end{aligned}
$$

where for $j<d, \boldsymbol{n}^{j}=\left(n_{j}, \ldots, n_{d-1}\right),\left|\boldsymbol{n}^{j}\right|=n_{j}+\cdots+n_{d-1}$ and for notational convenience $\left|\boldsymbol{n}^{d}\right|=0$.

Proposition 2. The conditional binomial construction of multidimensional Krawtchouk polynomials, Xu (2013), is a special case of the multidimensional Krawtchouk polynomials where the orthogonal basis is the (unscaled) Irwin-Lancaster basis: $u^{(0)}=1$ and

$$
u_{k}^{(j)}= \begin{cases}0 & k<j, \\ -\left(1-\left|\boldsymbol{p}_{j}\right|\right) / p_{j} & k=j, \\ 1 & k=j+1, \ldots, d .\end{cases}
$$

for $j=1, \ldots, d-1 ; k=1, \ldots, d$. 
Proof. By lemma 1, it is sufficient to show that the leading terms in both sets of orthogonal polynomials are identical. Using the hypergeometric expansion

$$
\begin{aligned}
K_{n_{j}}\left(x_{j} ;\right. & \left.\frac{p_{j}}{1-\left|\boldsymbol{p}_{j-1}\right|}, N-\left|\boldsymbol{x}_{j-1}\right|-\left|\boldsymbol{n}^{j+1}\right|\right)\left(-N+\left|\boldsymbol{x}_{j-1}\right|+\left|\boldsymbol{n}^{j+1}\right|\right)_{\left(n_{j}\right)} \\
=\sum_{k=0}^{n_{j}} & \frac{\left(-n_{j}\right)_{(k)}\left(-x_{j}\right)_{(k)}}{\left(-N+\left|\boldsymbol{x}_{j-1}\right|+\left|\boldsymbol{n}^{j+1}\right|\right)_{(k)}}\left(\frac{1-\left|\boldsymbol{p}_{j-1}\right|}{p_{j}}\right)^{k} \frac{1}{k !} \\
& \times\left(-N+\left|\boldsymbol{x}_{j-1}\right|+\left|\boldsymbol{n}^{j+1}\right|\right)_{\left(n_{j}\right)} .
\end{aligned}
$$

The leading coefficient in

$$
\begin{aligned}
& \frac{\left(-N+\left|\boldsymbol{x}_{j-1}\right|+\left|\boldsymbol{n}^{j+1}\right|\right)_{\left(n_{j}\right)}}{\left(-N+\left|\boldsymbol{x}_{j-1}\right|+\left|\boldsymbol{n}^{j+1}\right|\right)_{(k)}} \\
& \quad=\left(-N+\left|\boldsymbol{x}_{j-1}\right|+\left|\boldsymbol{n}^{j+1}\right|+k\right) \cdots\left(-N+\left|\boldsymbol{x}_{j-1}\right|+\left|\boldsymbol{n}^{j+1}\right|+n_{j}-1\right)
\end{aligned}
$$

is

$$
\left(-N+\left|\boldsymbol{x}_{j-1}\right|\right)^{n_{j}-k} .
$$

The leading coefficient in (11) is therefore

$$
\begin{aligned}
& \sum_{k=0}^{n_{j}}\left(-n_{j}\right)_{(k)}\left(-x_{j}\right)^{k}\left(\frac{1-\left|\boldsymbol{p}_{j-1}\right|}{p_{j}}\right)^{k}\left(-N+\left|\boldsymbol{x}_{j-1}\right|\right)^{n_{j}-k} \frac{1}{k !} \\
& \quad=(-1)^{n_{j}}\left(-x_{j} \frac{1-\left|\boldsymbol{p}_{j-1}\right|}{p_{j}}+N-\left|\boldsymbol{x}_{j-1}\right|\right)^{n_{j}} \\
& \quad=(-1)^{n_{j}}\left(-x_{j} \frac{1-\left|\boldsymbol{p}_{j}\right|}{p_{j}}+x_{j+1}+\cdots+x_{d}\right)^{n_{j}} \\
& \quad=(-1)^{n_{j}}\left(\sum_{k=1}^{d} u_{k}^{(j)} x_{k}\right)^{n_{j}} .
\end{aligned}
$$

The leading coefficient in $K_{\boldsymbol{n}}(\boldsymbol{x} ; \boldsymbol{p}, N)$ is then seen to be

$$
\frac{1}{(-N)_{(|\boldsymbol{n}|)}} \prod_{j=1}^{d-1} \frac{p_{j}^{n_{j}}}{\left(1-\left|\boldsymbol{p}_{j-1}\right|\right)^{n_{j}}}\left(\sum_{k=1}^{d} u_{k}^{(j)} x_{k}\right)^{n_{j}}
$$

which completes the proof. 
We now calculate the proportionality constants in the two systems. If $\boldsymbol{x}=N \boldsymbol{e}_{d}$ the generating function (44) for the multivariate Krawtchouk polynomials is

$$
\left(1+\sum_{j=1}^{d-1} u_{d}^{(j)} w_{j}\right)^{N}=\left(1+\sum_{j=1}^{d-1} w_{j}\right)^{N}
$$

so

$$
Q_{n}\left(N \boldsymbol{e}_{d}, \boldsymbol{u}\right)=\frac{N !}{(N-|\boldsymbol{n}|) ! \prod_{j=1}^{d-1} n_{j} !}
$$

As a comparison

$$
\begin{aligned}
K_{\boldsymbol{n}}\left(N \boldsymbol{e}_{d} ; \boldsymbol{p}, N\right) & =\frac{(-1)^{|\boldsymbol{n}|}}{(-N)_{(|\boldsymbol{n}|)}} \prod_{j=1}^{d-1} \frac{p_{j}^{n_{j}}}{\left(1-\left|\boldsymbol{p}_{j-1}\right|\right)^{n_{j}}}\left(-N+\left|\boldsymbol{n}^{j+1}\right|\right)_{\left(n_{j}\right)} \\
& =(-1)^{|\boldsymbol{n}|} \prod_{j=1}^{d-1}\left(\frac{p_{j}}{1-\left|\boldsymbol{p}_{j-1}\right|}\right)^{n_{j}}
\end{aligned}
$$

Comparing the two polynomials at $\boldsymbol{x}=N \boldsymbol{e}_{d+1}$ gives the next corollary.

\section{Corollary 1.}

$$
K_{\boldsymbol{n}}(\boldsymbol{x} ; \boldsymbol{p}, N)=\frac{1}{(-N)_{(|\boldsymbol{n}|)}} \prod_{j=1}^{d-1} n_{j} !\left(\frac{p_{j}}{1-\left|\boldsymbol{p}_{j-1}\right|}\right)^{n_{j}} Q_{\boldsymbol{n}}(\boldsymbol{x}, \boldsymbol{u}) .
$$

The generating function definition makes it easy to compute various transforms of $Q_{n}$ as a product of linear forms

$$
\mathbb{E}\left[\prod_{i=1}^{d} \phi_{i}^{X} Q_{\boldsymbol{n}}(X)\right]=\left(\begin{array}{c}
N \\
|\boldsymbol{n}|
\end{array}\right)\left(\begin{array}{c}
|\boldsymbol{n}| \\
\boldsymbol{n}
\end{array}\right) T_{0}(\phi)^{N-|\boldsymbol{n}|} T_{1}(\phi)^{n_{1}} \cdots T_{d-1}(\phi)^{n_{d-1}}
$$

where

$$
T_{i}(\phi)=\sum_{j=1}^{d} \phi_{j} p_{j} u_{j}^{(i)}, 0 \leq i \leq d-1 .
$$

Using random variable notation can provide elegant formulae. Let $Z_{1}, \ldots, Z_{N}$ be independent identically distributed random variables with $P(Z=k)=$ $p_{k}, 1 \leq k \leq d$. Set $X_{i}=\left|\left\{k: Z_{k}=i\right\}\right|$ so $\left(X_{1}, \ldots, X_{d}\right)$ has a $m(\boldsymbol{x}, \boldsymbol{p})$ distribution. Then (6) gives (with both sides random variables)

$$
G(\boldsymbol{X} ; \boldsymbol{w} ; \boldsymbol{u})=\prod_{k=1}^{N}\left(1+\sum_{l=1}^{d-1} w_{l} v_{Z_{k}}^{(l)}\right)
$$


Expanding the right hand side gives

$$
Q_{\boldsymbol{n}}(\boldsymbol{X}, \boldsymbol{u})=\sum_{\left\{A_{l}\right\}} \prod_{k_{1} \in A_{1}} u_{Z_{k_{1}}}^{(1)} \ldots \prod_{k_{d-1} \in A_{d-1}} u_{Z_{k_{d-1}}}^{(d-1)}
$$

where the summation is over all partitions of $N$ into subsets $\left\{A_{l}\right\}$ such that $\left|A_{l}\right|=n_{l}, l=1, \ldots, d-1$. This shows that $Q_{\boldsymbol{n}}(\boldsymbol{x}, \boldsymbol{u})$ is a polynomial of degree $n-|\boldsymbol{n}|$ in $\left(S_{1}(\boldsymbol{x}), \ldots, S_{d-1}(\boldsymbol{x})\right)$ with

$$
S_{i}(\boldsymbol{x})=\sum_{j=1}^{d} u_{j}^{(l)} x_{j}, i=1, \ldots, d-1
$$

whose only term of maximal degree is $\prod_{k=1}^{d-1} S_{k}^{n_{k}}(\boldsymbol{x})$.

General 1-dimensional orthogonal polynomials $\left\{P_{n}(x)\right\}$ satisfy a three term recurrence for $x P_{n}(x)$. A similar recurrence holds for $S_{i}(\boldsymbol{x}) Q_{\boldsymbol{n}}(\boldsymbol{x} ; \boldsymbol{u})$. Scale $Q_{\boldsymbol{n}}(\boldsymbol{x} ; \boldsymbol{u})$ so that the leading coefficient of $\prod_{i=1}^{d-1} S_{i}(\boldsymbol{x})^{n_{i}}$ is unity by taking $Q_{\boldsymbol{n}}^{*}(\boldsymbol{x} ; \boldsymbol{u})=\prod_{i=1}^{d-1} n_{i} ! Q_{\boldsymbol{n}}(\boldsymbol{x} ; \boldsymbol{u})$. Let $c(i, l, k)=\sum_{j=1}^{d} u_{j}^{(i)} u_{j}^{(l)} u_{j}^{(k)} p_{j}$. Then a generating function argument shows that

$$
\begin{array}{r}
S_{i}(\boldsymbol{x}) Q_{\boldsymbol{n}}^{*}(\boldsymbol{x}, \boldsymbol{u})=Q_{\boldsymbol{n}+\boldsymbol{e}_{i}}^{*}(\boldsymbol{x}, \boldsymbol{u})+(N-|n|+1) Q_{\boldsymbol{n}-\boldsymbol{e}_{i}}^{*}(\boldsymbol{x}, \boldsymbol{u}) \\
+\sum_{l, k=1}^{d-1} c(i, l, k) Q_{\boldsymbol{n}-\boldsymbol{e}_{l}+\boldsymbol{e}_{k}}^{*}(\boldsymbol{x}, \boldsymbol{u}) .
\end{array}
$$

\subsection{Three Examples}

This section develops three detailed examples; the first gives an 'always available' basis $\left\{u^{(l)}\right\}$ for general $\boldsymbol{p}$. This turns out to diagonize a Metropolis algorithm and satisfy a hypergroup property developed further in section 2.4. The second involves group characters and 'explains' the hypergroup nomenclature; the third is a development from physics. It offers ways of generalizing the construction of section 2.1 to general space.

Example 2.1 One simple closed form choice of $\left\{u^{(l)}\right\}$ is given by IrwinHelmert matrices (Lancaster, 1969). The basis is a scaled version of (10). Given $\boldsymbol{p}$, let $a_{i}^{2}=p_{i}, A_{i}^{2}=p_{d}+\cdots+p_{i}$. Define a $d \times d$ matrix $\mathcal{U}$ with first row $(1, \ldots, 1)$ and $i^{\text {th }}$ row

$$
(\overbrace{0, \ldots, 0}^{d-i}, \frac{-A_{d+2-i}}{a_{d+1-i} A_{d+1-i}}, \frac{a_{d+1-i}}{A_{d+2-i} A_{d+1-i}}, \ldots, \frac{a_{d+1-i}}{A_{d+2-i} A_{d+1-i}}), 2 \leq i \leq d .
$$


Thus when $d=5, \mathcal{U}$ is

$$
\left[\begin{array}{ccccc}
1 & 1 & 1 & 1 & 1 \\
0 & 0 & 0 & -\frac{A_{5}}{a_{4} A_{4}} & \frac{a_{4}}{A_{4} A_{5}} \\
0 & 0 & -\frac{A_{4}}{a_{3} A_{3}} & \frac{a_{3}}{A_{3} A_{4}} & \frac{a_{3}}{A_{3} A_{4}} \\
0 & -\frac{A_{3}}{a_{2} A_{2}} & \frac{a_{2}}{A_{2} A_{3}} & \frac{a_{2}}{A_{2} A_{3}} & \frac{a_{2}}{A_{2} A_{3}} \\
-\frac{A_{2}}{a_{1}} & \frac{a_{1}}{A_{1} A_{2}} & \frac{a_{1}}{A_{1} A_{2}} & \frac{a_{1}}{A_{1} A_{2}} & \frac{a_{1}}{A_{1} A_{2}}
\end{array}\right]
$$

The rows of $\mathcal{U}$ are an orthonormal basis. Thus

$$
u_{j}^{(0)} \equiv 1, u_{j}^{(i)}= \begin{cases}0 & 1 \leq j \leq i-1 \\ -\frac{A_{i+1}}{a_{i} A_{i}} & j=i \\ \frac{a_{i}}{A_{i} A_{i+1}} & j>i .\end{cases}
$$

These matrices were used by Lancaster and Irwin to decompose the usual chi-square test for goodness of fit to a multinomial model into $d$ orthogonal pieces. See Lancaster (1969). Saltzman (2010) has observed that the $u^{(l)}$ diagonalize a natural Markov chain. We state this formally:

Proposition 3. (Saltzman) Let $p_{1} \geq \cdots \geq p_{d}$ be a probability distribution on $[d]=\{1,2, \ldots, d\}$ and $u^{(l)}$ be defined by (17). Let $K(i, j)$ be the 'random scan Metropolis' Markov chain on [d]: From $i$, pick $j$ uniformly in [d]. If $j \leq i$, move to $j$. If $j>i$, flip a $p_{j} / p_{i}$ coin. If heads, move to $j$; else stay at $i$. This is a reversible Markov chain with stationary distribution $\boldsymbol{p}$. It has $u^{(l)}$ as (right) eigenfunctions: $K u^{(0)}=u^{(0)}$ and $K u^{(l)}=\beta_{l} u^{(l)}$ with

$$
\beta_{l}=1-\frac{A_{l}^{2}}{d a_{l}^{2}}, 1 \leq l \leq d-1
$$

Proof The diagonalization of the random scan Metropolis chain is a special case of a theorem of Liu (1996). His result gives similar eigen-values and eigen-vectors for the Metropolis chain with general proposal.

The hypergroup property of an orthogonal basis $\left\{u^{(l)}\right\}$ allows delineation of all Markov chains and all (Lancaster) bivariate distributions admiting $\left\{u^{(l)}\right\}$ as eigen-bases. Section 2.4 below shows that the multivariate Krawtchouk polynomials satisfy the hypergroup property provided the underlying $\left\{u^{(l)}\right\}$ do. To provide examples, we now give a necessary and sufficient condition on the Irwin-Helmert basis for this property. 
One way to state the property is to transform $\left\{u^{(l)}\right\}$ into an orthogonal matrix $H$ by multiplying each column by $p_{j}^{1 / 2}$; thus suppose $\left\{u^{(l)}\right\}$ satisfies (2.2) and define

$$
H_{i j}=u_{j}^{(i-1)} p_{j}^{1 / 2} \text { for } i, j \in[d] \text {. (Thus } h_{1 j}=p_{j}^{1 / 2} \text { for } j \in[d] \text { ). }
$$

For a general orthogonal $H$, the hypergroup property is

$$
s(j, k, l)=\sum_{i=1}^{d} h_{i j} h_{i k} h_{i l} / h_{i d} \geq 0, \text { for all } j, k, l \in[d] .
$$

For this to be defined, $h_{i d} \neq 0$ for $i \in[d]$. This property is equivalent to the usual conception of a hypergroup if we take (without loss of generality) $h_{i d}>0$ because

$$
h_{i j} h_{i k}=h_{i d} \sum_{l=1}^{d} s(j, k, l) h_{i l} .
$$

That is, the product $h_{i j} h_{i k}$ can be expressed as a linear combination of terms $h_{i l}$ with non-negative coefficients. The property (19) is satisfied by the Irwin-Helmert matrices and will be assumed throughout this section. A familiar example, with $d=4$ and $p=\left(\frac{1}{4}, \frac{1}{4}, \frac{1}{4}, \frac{1}{4}\right)$ is

$$
H=\frac{1}{2}\left[\begin{array}{rrrr}
1 & 1 & 1 & 1 \\
-1 & 1 & -1 & 1 \\
1 & 1 & -1 & -1 \\
-1 & 1 & 1 & -1
\end{array}\right]
$$

However the reader will find it impossible to construct a $3 \times 3$ orthogonal $H$ satisfying (19) with first row $\left(\frac{1}{\sqrt{3}}, \frac{1}{\sqrt{3}}, \frac{1}{\sqrt{3}}\right)$. Indeed Bakry and Huet (2008) show that a $d \times d$ orthogonal $H$ satisfying (19) with constant first row exists if and only if $d=2^{k}$ for some $k \geq 1$. This raises the question: what $\left(p_{1}, \ldots, p_{d}\right)$ admit $u^{(l)}$ and $H$ satisfying (19)? The proposition below gives examples if $\boldsymbol{p}$ satisfies a monotonicity property. Say that $\boldsymbol{p}$ is strongly monotone if

$$
p_{d} \leq p_{d-1}, p_{d}+p_{d-1} \leq p_{d-2}, \ldots, p_{d}+\cdots+p_{2} \leq p_{1} .
$$

For example when $d=3,\left(\frac{1}{2}, \frac{1}{3}, \frac{1}{6}\right)$ and $\left(\frac{1}{2}, \frac{1}{4}, \frac{1}{4}\right)$ are strongly monotone.

Proposition 4. Suppose that $\boldsymbol{p}$ is strongly monotone. For the Irwin-Helmet matrix (17) define an orthogonal matrix $H$ by (18). Then $H$ satisfies the hypergroup property. Further the strongly monotone $\boldsymbol{p}$ form a full dimensional (dimension $d-1$ ) compact-convex simplex with extreme points

$$
(0, \ldots, 0,1),\left(0, \ldots, 0, \frac{1}{2}, \frac{1}{2}\right),\left(0, \ldots, 0, \frac{1}{4}, \frac{1}{4}, \frac{1}{2}\right), \ldots,\left(\frac{1}{2^{d-1}}, \frac{1}{2^{d-1}}, \frac{1}{2^{d-2}}, \ldots, \frac{1}{2}\right) .
$$


Proof It may help the reader to have an example. For $d=5, a_{i}^{2}=p_{i}$, $A_{i}^{2}=p_{d}+p_{d-1}+\cdots+p_{i},\left(\right.$ so $\left.A_{1}=1\right)$,

$$
H=\left[\begin{array}{ccccc}
a_{1} & a_{2} & a_{3} & a_{4} & a_{5} \\
0 & 0 & 0 & -\frac{A_{5}}{A_{4}} & \frac{a_{4} a_{5}}{A_{4} A_{5}} \\
0 & 0 & -\frac{A_{4}}{A_{3}} & \frac{a_{3} a_{4}}{A_{3} A_{4}} & \frac{a_{3} a_{5}}{A_{3} A_{4}} \\
0 & -\frac{A_{3}}{A_{2}} & \frac{a_{2} a_{3}}{A_{2} A_{3}} & \frac{a_{2} a_{4}}{A_{2} A_{3}} & \frac{a_{2} a_{5}}{A_{2} A_{3}} \\
-A_{2} & \frac{a_{1} a_{2}}{A_{2}} & \frac{a_{1} a_{3}}{A_{2}} & \frac{a_{1} a_{4}}{A_{2}} & \frac{a_{1} a_{5}}{A_{2}}
\end{array}\right] .
$$

Observe that $s(j, k, l)$ is invariant under permutation of indices, so without loss of generality take $j \leq k \leq l$. The following argument shows that

$$
\begin{aligned}
& s(j, k, l) \geq 0 \text { if any of } j, k, l=d, \\
& \quad \text { indeed } s(j, j, d)=s(d, d, d)=1, s(j, d, d)=s(j, k, d)=0 ; \\
& s(i, i, j) \geq 0 \\
& s(i, j, j)=0 ; \\
& s(j, k, l)=0 \text { for } 1 \leq j<k<l<d .
\end{aligned}
$$

Inequalities (23)-(26) hold for any $a_{i}$. Strong monotonicity is not needed. This covers all choices except $s(i, i, i), i \in[d]$. The positivity of these quantities is in a one to one correspondence with the linear inequalities $p_{d}+\cdots+p_{i+1} \leq p_{i}, 1 \leq i \leq d-1$. To prove (23) observe eg $s(j, k, d)=$ $\sum_{i=1}^{d} h_{i j} h_{i k}=\delta_{j k} \geq 0$. The other cases are similar. Thus without loss of generality $1 \leq j \leq k \leq l<d$ for the rest of the proof. For (24), observe that for $i<j, s(i, i, j)$ is the sum of positive terms.

The proof of (25) is by induction on $d$. It is basic to check for $d=2,3$. For larger $d$, first consider

$$
s(1, j, j)=\frac{a_{1} a_{j}^{2}}{a_{d}}-A_{2}\left(\frac{a_{1} a_{j}}{A_{2}}\right)^{2} \frac{A_{2}}{a_{1} a_{d}}=0 .
$$

Next consider the matrix $H$ with the first column and last row deleted. This is a $(d-1) \times(d-1)$ matrix of the same form with the first row divided by $A_{2}$. By induction

$$
\frac{p_{d}}{A_{2}} \leq \frac{p_{d-1}}{A_{2}}, \frac{p_{d}+p_{d-1}}{A_{2}} \leq \frac{p_{d-2}}{A_{2}}, \ldots, \frac{p_{d}+\cdots+p_{j}}{A_{2}} \leq \frac{p_{2}}{A_{2}} .
$$

This finishes the proof of the hypergroup property. The final claims are about the set of strongly monotone probabilities. Observe that $p_{d}+\cdots+$ 
$p_{2} \leq p_{1}$ is equivalent to $p_{1} \geq \frac{1}{2}$. From this $p_{2} \leq \frac{1}{2}$. Also $p_{2}+p_{3} \leq \frac{1}{2}$. Along with $p_{3} \leq p_{2}$, this gives $p_{2} \leq \frac{1}{4}$. Continuing gives $p_{i} \leq \frac{1}{2^{i}}, 2 \leq$ $i \leq d$. From this the claimed extreme points are all extreme. They can be seen to be all of the extreme points with unique representation by a similar greedy algorithm. Given strongly monotone $\left(p_{1}, \ldots, p_{d}\right)$, subtract off $p_{d} 2^{d-1}\left(\frac{1}{2^{d-1}}, \frac{1}{2^{d-1}}, \ldots, \frac{1}{2}\right)$. The difference is positive and normalizing gives a strictly monotone probability with the first entry zero. Continuing gives $\boldsymbol{p}$ as a linear combination of extreme points. The representation is unique because the extreme points

$(1,0,0, \ldots, 0),\left(\frac{1}{2}, \frac{1}{2}, 0, \ldots, 0\right),\left(\frac{1}{2}, \frac{1}{4}, \frac{1}{4}, 0, \ldots, 0\right), \ldots,\left(\frac{1}{2}, \frac{1}{4}, \frac{1}{8}, \ldots, \frac{1}{2^{d-1}}, \frac{1}{2^{d-1}}\right)$

are linearly independent.

Proposition 4 suggests a question about the Irwin-Lancaster bases. $\left\{u^{(l)}\right\}$ posesses the GKS property, Bakry and Huet (2008), if for all $l, r$

$$
u^{(l)} u^{(r)}=\sum_{k} c_{l r}^{k} u^{(k)}
$$

where $c_{l r}^{k} \geq 0$ for $l, r, k=0, \ldots, d-1$. The GKS property insures a variety of probabilistic inequalities hold. Do the Irwin-Helmert bases (17) satisfy GKS under the assumptions of Proposition 4? The next proposition affirms that this is true.

Proposition 5. The Irwin-Lancaster bases possess the GKS property if and only if $\boldsymbol{p}$ is strongly monotone.

Proof The GKS property holds if and only if for $0 \leq l \leq m \leq d-1$

$$
\sum_{j=1}^{d} u_{j}^{(l)} u_{j}^{(m)} u_{j}^{(r)} p_{j} \geq 0
$$

It is convenient to define

$$
\begin{aligned}
v_{j}^{(r)} & =\frac{A_{r} A_{r+1}}{a_{r}} u_{j}^{(r)} \\
& = \begin{cases}-\frac{p_{r+1}+\cdots+p_{d}}{p_{r}} & \text { if } j=r \\
1 & \text { if } j>r .\end{cases}
\end{aligned}
$$


Then (27) holding is equivalent to

$$
c(l, m, r) \stackrel{\text { Def }}{=} \sum_{j=1}^{d} v_{j}^{(l)} v_{j}^{(m)} v_{j}^{(r)} p_{j} \geq 0 .
$$

Evaluating the triple sum

$$
c(l, m, r)= \begin{cases}-\frac{p_{r+1}+\cdots+p_{d}}{p_{r}} p_{r}+p_{r+1}+\cdots+p_{d}=0, & \text { if } l \leq m<r \\ \frac{\left(p_{r+1}+\cdots+p_{d}\right)^{2}}{p_{r}^{2}} p_{r}+p_{r+1}+\cdots+p_{d} \geq 0, & \text { if } l<m=r \\ \frac{\left(p_{r+1}+\cdots+p_{d}\right)}{p_{r}^{2}}\left(p_{r}^{2}-\left(p_{r+1}+\cdots+p_{d}\right)^{2}\right) \geq 0, & \text { if } l=m=r .\end{cases}
$$

Positivity in the last case holds if and only if $\boldsymbol{p}$ is strongly monotone.

Hypergroups for Groups. Let $\mathcal{G}$ be a finite group with conjugacy classes $\mathcal{C}_{1}, \mathcal{C}_{2}, \ldots, \mathcal{C}_{d}$ and irreducible characters $\chi_{1}, \ldots, \chi_{d}$. Label those so that $\mathcal{C}_{d}=\{\mathrm{id}\}, \chi_{1} \equiv 1$ (the trivial character). Background can be found in James and Liebeck (2001), Issacs (1994), Diaconis (1988). Define $p_{i}=$ $\left|\mathcal{C}_{i}\right| /|\mathcal{G}|$, for $i=1, \ldots, d$. It is a classical fact that

$$
\sum_{i} \chi_{i}\left(\mathcal{C}_{j}\right) \chi_{i}\left(\mathcal{C}_{k}\right) \chi_{i}\left(\mathcal{C}_{l}\right) \geq 0 \text { for all } j, k, l \in[d]
$$

see eg Bakry and Huet (2008), Proposition 2.6. This implies

Proposition 6. Let $\mathcal{G}$ be a finite group with $d$ conjugacy classes. Suppose that all of the characters are real valued. Then the matrix $H$

$$
h_{i j}=\chi_{i}\left(\mathcal{C}_{j}\right) \sqrt{p_{j}},\left(p_{j}=\left|\mathcal{C}_{j}\right| /|\mathcal{G}|\right)
$$

is orthogonal and satisfies the hypergroup and GKS properties.

Example Let $\mathcal{G}=\mathcal{C}_{2}^{n}$, the group of binary $n$-tuples under commutative addition. This is an abelian group so the conjugacy classes are single points. For $x \in \mathcal{G}$, let $\chi_{x}(y)=(-1)^{x \cdot y} \cdot\left\{\chi_{x}\right\}_{x \in \mathcal{G}}$ are the irreducible characters and $\mathcal{G}$ is real. Here $|\mathcal{G}|=2^{n}$, and $p_{i}=1 / 2^{n}$. When $n=2$ the relevant basis is displayed in (20).

Example Let $\mathcal{G}=S_{3}$, the symmetric group. There are three conjugacy classes

$$
\mathcal{C}_{1}=\{(1,2),(1,3),(2,3)\}, \mathcal{C}_{2}=\{(1,2,3),(1,3,2)\}, \mathcal{C}_{3}=\{\operatorname{id}\} .
$$


Thus $p_{1}=\frac{1}{2}, p_{2}=\frac{1}{3}, p_{3}=\frac{1}{6}$. The character table and associated $H$ are (bordered rows and columns)

$$
\chi_{1} \chi_{2} \chi_{3}\left[\begin{array}{rrr}
\mathcal{C}_{1} & \mathcal{C}_{2} & \mathcal{C}_{3} \\
1 & 1 & 1 \\
0 & -1 & 2 \\
-1 & 1 & 1
\end{array}\right] \quad H=\left[\begin{array}{rrr}
\frac{1}{\sqrt{2}} & \frac{1}{\sqrt{3}} & \frac{1}{\sqrt{6}} \\
0 & -\frac{1}{\sqrt{3}} & \frac{2}{\sqrt{6}} \\
-\frac{1}{\sqrt{2}} & \frac{1}{\sqrt{3}} & \frac{1}{\sqrt{6}}
\end{array}\right] .
$$

The orthogonal matrices $H$ in these examples also have a probabilistic interpretation; they are the eigenvectors of any random walk on $\mathcal{G}$ which is constant on conjugacy classes, see Diaconis (1988), chapter 3.

Example Bosonic Fock space and second quantization.

The multivariate Krawtchouk polynomials are closely related to a basic construction in modern physics. This connection illuminates the construction, suggesting natural generalizations to infinite spaces and to varying numbers of particles. Good references for physics are Desai (2010), chapter 3, Reed and Simon (1975), chapter X.7 and Feynman (1972), chapter 6.7.

We begin with the general story - symmetrized tensors, then symmetrized to the multinomial, finally discussions and generalizations.

Let $V$ be a vector space $V^{\otimes N}$ the $N$-fold tensor product and $V_{S}^{\otimes N}$ the elements in $V^{\otimes N}$ invariant under the symmetric group $S_{N}$. If $<\cdot|\cdot\rangle$ is an inner product on $V$ then $V^{\otimes N}$ becomes an inner product space with $<a_{1} \otimes \cdots \otimes a_{N}\left|b_{1} \otimes \cdots \otimes b_{N}>=<a_{1}\right| b_{1}>\cdots<a_{N} \mid b_{N}>$. Further, $V_{S}^{\otimes N}$ inherits an inner product. If $\overline{a_{1} \otimes \cdots \otimes a_{N}}$ denotes symmetrization,

$$
<\overline{a_{1} \otimes \cdots \otimes a_{N}} \mid \overline{b_{1} \otimes \cdots \otimes b_{N}}>=\operatorname{per}\left[\begin{array}{c}
<a_{1}\left|b_{1}>\ldots,<a_{1}\right| b_{N}> \\
\ldots \\
<a_{N}\left|b_{1}>\ldots,<a_{N}\right| b_{N}>
\end{array}\right],
$$

Feynman (1972), (6.3). If $\left\{u^{(l)}\right\}_{l \in \mathcal{L}}$ is an orthogonal basis for $V(\operatorname{dim} V=\infty$ is allowed, ) then $V^{\otimes N}$ has $u^{\left(l_{1}\right)} \otimes \cdots \otimes u^{\left(l_{N}\right)}=u^{(l)}$ as an orthogonal basis with $l_{i} \in \mathcal{L}$. Symmetrizing these gives

$$
\bar{u}^{(l)}=\sum_{\sigma \in S_{N}} u^{\left(l_{\sigma 1}\right)} \otimes \cdots \otimes u^{\left(l_{\sigma N}\right)} .
$$

Because of the symmetry, $\bar{u}^{(l)}$ only depends on $n_{i_{1}}, n_{i_{2}}, \ldots$, with $n_{l}$ the number of $i$ such that $l_{i}=l$. The $\bar{u}^{(l)}$ are an orthonormal basis for $V_{S}^{\otimes w}$. In the physics literature they are often denoted by $\mid n_{1}, n_{2} \cdots>$. Specialize to 
the case where $V=L^{2}(\mu)$ for $\mu$ a probability measure on $(\mathfrak{X}, \mathcal{B})$. Let $\left\{u^{(l)}\right\}$ be an orthogonal basis with $u^{(0)}(x) \equiv 1$. The basis elements are

$$
\bar{f}^{(\boldsymbol{l})}\left(y_{1}, y_{2}, \ldots, y_{N}\right)=\sum_{\sigma \in S_{N}} \prod_{i=1}^{N} f^{\left(l_{i}\right)}\left(y_{\sigma(i)}\right) .
$$

The degree of $f^{(\boldsymbol{n})}$ is $|\boldsymbol{n}|=n_{1}+n_{2}+\cdots$. Thus the degree 0 element is

$$
\bar{f}^{(0)} \equiv 1 \text {. }
$$

The degree one basis vectors are (up to the proportionality constant $(N-1)$ !)

$$
\bar{f}^{(l)}\left(y_{1}, \ldots, y_{N}\right)=\sum_{\sigma \in S_{N}} f^{\left(l_{i}\right)}\left(y_{\sigma(i)}\right)
$$

Now suppose that the underlying space $\mathfrak{X}=[d]$ with $\mu=\boldsymbol{P}$. If $\left\{u^{(l)}\right\}$ is chosen as in (2) and $z_{1}, \ldots, z_{N}$ has $i$ appearing $x_{i}(\boldsymbol{z})$ times

$$
\bar{f}^{\left(e_{l}\right)}=\sum_{j=1}^{d} u_{j}^{(l)} x_{j}, \quad 1 \leq l \leq d-1 .
$$

These are the linear Krawtchouk polynomials. Similarly, the higher degree basis terms are the multivariate Krawtchouk polynomials.

In the physics literature if $A$ is a self adjoint operator on $V$, then

$$
(A \otimes I \otimes \cdots \otimes I)+(I \otimes A \otimes \cdots \otimes)+\cdots+(I \otimes I \otimes \cdots \otimes A)
$$

operates on $V_{S}^{\otimes N}$. It is called the second quantization of $A$. If $V=L^{2}(\mu)$ is our $d$-dimensional space and $A$ is the transition matrix of a $p$-reversible Markov chain, the second quantization (divided by $N$ ) is just "pick a coordinate at random, if it is colour $i$ change it to $j$ with probability $A(i, j)$ ". See Feynman (1972), section 6.8, for the physics version.

The development above shows how to generalize from $[d]$ to a general space. The physics development has an additional feature; the creation and destruction operators $a^{+}(\cdot)$ and $a^{-}(\cdot)$. These translate into "add or subtract a ball" in the multinomial picture. They necessitate working in the enhanced state space $\bigoplus_{N=0}^{\infty} V_{S}^{\otimes N}$ (Bosonic Fock space). We will not develop the story further, but believe there is a lot to be done translating between fields. 


\subsection{Self Duality}

The univariate Krawtchouk polynomials $Q_{n}$ satisfy the useful duality equation $Q_{n}(x)=Q_{x}(n)$. This section defines a $d$-dimensional extension, used in section 2.4 to prove the hypergroup property which is a crucial ingredient in section three.

Duality is the easiest to describe by considering a more general class of polynomials $\widehat{Q}_{\boldsymbol{n}^{+}}(\boldsymbol{x}, H)$, where $H$ is a $d \times d$ orthogonal matrix, $\boldsymbol{n}^{+}=$ $\left(n_{1}^{+}, \ldots, n_{d}^{+}\right)$with $\left|\boldsymbol{n}^{+}\right|=N, \boldsymbol{x}=\left(x_{1}, \ldots, x_{d}\right)$ has $|\boldsymbol{x}|=N$. Define $\widehat{Q}_{\boldsymbol{n}^{+}}(\boldsymbol{x}, H)$ as the coefficient of

$$
\left(\begin{array}{l}
N \\
\boldsymbol{n}^{+}
\end{array}\right) \prod_{j=1}^{d} w_{j}^{n_{j}^{+}}\left(\begin{array}{l}
N \\
\boldsymbol{x}
\end{array}\right) \prod_{i=1}^{d} z_{i}^{x_{i}}
$$

in

$$
\left[\sum_{i, j=1}^{d} h_{i j} w_{i} z_{j}\right]^{N}
$$

$\widehat{Q}_{\boldsymbol{n}^{+}}(\boldsymbol{x}, H)$ is also the coefficient of

$$
\left(\begin{array}{c}
N \\
n^{+}
\end{array}\right) \prod_{j=1}^{d} w_{j}^{n_{j}^{+}} \text {in } \prod_{j=1}^{d}\left\{\sum_{i=1}^{d} h_{i j} w_{i}\right\}^{x_{i}} .
$$

or the coefficient of

$$
\left(\begin{array}{l}
N \\
\boldsymbol{x}
\end{array}\right) \prod_{i=1}^{d} z_{i}^{x_{j}} \text { in } \prod_{i=1}^{d}\left\{\sum_{j=1}^{d} h_{i j} z_{j}\right\}^{n_{i}^{+}} .
$$

There is an evident duality

$$
\widehat{Q}_{\boldsymbol{n}^{+}}(\boldsymbol{x}, H)=\widehat{Q}_{\boldsymbol{x}}\left(\boldsymbol{n}^{+}, H^{T}\right),
$$

where $H^{T}$ denotes the transpose of $H$. The generating function (32) is a generating function for both systems in (33). The variable is $\boldsymbol{x}$ with index $\boldsymbol{n}^{+}$for the system on the left side, and the variable is $\boldsymbol{n}^{+}$with index $\boldsymbol{x}$ for the dual system on the right side. To make the connection to Krawtchouk polynomials for $\left\{u^{(l)}\right\}$ set

$$
h_{i j}=u_{j}^{(i-1)} p_{j}^{1 / 2}, i, j \in\{1, \ldots, d\}, \quad \text { so } h_{i j}=p_{j}^{1 / 2},
$$

and

$$
\boldsymbol{n}^{+}=\left(N-|\boldsymbol{n}|, n_{1}, \ldots, n_{d-1}\right),|\boldsymbol{n}| \leq N .
$$


Then

$$
\widehat{Q}_{\boldsymbol{n}^{+}}(\boldsymbol{x}, H)=\left(\begin{array}{l}
N \\
\boldsymbol{n}^{+}
\end{array}\right)^{-1} Q_{\boldsymbol{n}}(\boldsymbol{x}, \boldsymbol{v}) \prod_{j=1}^{d} p_{j}^{x_{j} / 2} .
$$

with $H$ from (34). $\left\{\widehat{Q}_{\boldsymbol{n}^{+}}\right\}$satisfy the orthogonality relations

$$
\begin{aligned}
\sum_{\boldsymbol{x}} \widehat{Q}_{\boldsymbol{m}^{+}}(\boldsymbol{x}, H) \widehat{Q}_{\boldsymbol{n}^{+}}(\boldsymbol{x}, H)\left(\begin{array}{c}
N \\
\boldsymbol{x}
\end{array}\right) & =\delta_{\boldsymbol{m} \boldsymbol{n}}\left(\begin{array}{c}
N \\
\boldsymbol{n}^{+}
\end{array}\right)^{-1} \\
\sum_{\boldsymbol{n}^{+}} \widehat{Q}_{\boldsymbol{n}^{+}}\left(\boldsymbol{x}, H^{T}\right) \widehat{Q}_{\boldsymbol{n}^{+}}\left(\boldsymbol{y}, H^{T}\right)\left(\begin{array}{c}
N \\
\boldsymbol{n}^{+}
\end{array}\right) & =\delta_{\boldsymbol{x} \boldsymbol{y}}\left(\begin{array}{c}
N \\
\boldsymbol{x}
\end{array}\right)^{-1} .
\end{aligned}
$$

This shows that $\left\{\widehat{Q}_{n^{+}}\right\}$are orthogonal polynomials for the flat multinomial $\left\{p_{i}=1 / d\right\}$. A symmetrized product form is

$$
\widehat{Q}_{\boldsymbol{n}^{+}}(\boldsymbol{x}, H)=\left(\begin{array}{l}
N \\
\boldsymbol{n}^{+}
\end{array}\right)^{-1} \sum_{\left\{A_{l}\right\}_{l=1}^{d}} \prod_{k \in A_{1}} h_{1 z_{k}} \cdots \prod_{k \in A_{d}} h_{d z_{k}},
$$

where the summation is over all partitions of subsets of $\{1, \ldots, N\},\left\{A_{l}\right\}$ such that $\left|A_{l}\right|=n_{l}, l=1, \ldots, d$ with $\left\{z_{1}, \ldots, z_{N}\right\}$ a multi-set containing $x_{j}$ entries equal to $j, j=1, \ldots, d$.

\subsection{The Hypergroup Property}

Univariate Krawtchouk polynomials $Q_{n}(x)$ with $\mathbb{E}\left[Q_{m}(X) Q_{n}(X)\right]=\delta_{m n} h_{n}$ satisfy the hypergroup property

$$
\sum_{n=0}^{N} h_{n} Q_{n}(x) Q_{n}(y) Q_{n}(z) \geq 0, \text { for all } x, y, z=0,1, \ldots, N \text {. }
$$

This property was discovered and exploited by Eagleson (1969) in his solution of the Lancaster problem for the Binomial distribution. Vere-Jones (1971) and Dunkl and Ramirez (1974) study group theoretic properties of the hypergroup property of the Krawtchouk polynomials. In Diaconis and Griffiths (2012) the hypergroup property is used to characterize reversible Markov chains with (univariate) Krawtchouk polynomials as eigen-functions. Following Eagleson's work, a host of univariate orthogonal polynomials have been shown to satisfy the hypergroup property. A wonderful survey of this work is given by Bakry and Huet (2008). The purpose of this subsection 
is to study the hypergroup property for multivariate Krawtchouk polynomials. Applications are in section three. The main result shows that the hypergroup property is equivalent to a hypergroup property for the chosen underlying basis $\left\{u^{(l)}\right\}$. This allows the examples developed in section 2.2 to be used for the multinomial.

It is convenient to consider scaled multivariate Krawtchouk polynomials

$$
Q_{n}^{\diamond}(\boldsymbol{x}, \boldsymbol{u})=\frac{Q_{n}(\boldsymbol{x}, \boldsymbol{u})}{Q_{n}\left(N \boldsymbol{e}_{d}, \boldsymbol{u}\right)}
$$

Recall that $\boldsymbol{u}=\left\{u^{(0)}, \ldots, u^{(d-1)}\right\}$ is an orthonormal basis for functions on $[d]$ with respect to $\boldsymbol{p}$. The scaling gives $Q_{n}^{\diamond}\left(N \boldsymbol{e}_{d} ; \boldsymbol{u}\right)=1$ (the choice of coordinate $d$ in $e_{d}$ is chosen without loss of generality). From the generating function (4)

$$
Q_{\boldsymbol{n}}\left(N e_{d}, \boldsymbol{u}\right)=\left(\begin{array}{c}
N \\
\boldsymbol{n}^{+}
\end{array}\right) \prod_{i=1}^{d-1} b_{i}^{n_{i}}
$$

where

$$
b_{i}=u_{d}^{(i)} \neq 0
$$

is an assumption in force throughout. This is automatic if Irwin-Helmert matrices are used. In general

$$
\mathbb{E}\left[Q_{\boldsymbol{m}}^{\diamond}(\boldsymbol{X}, \boldsymbol{u}) Q_{\boldsymbol{n}}^{\diamond}(\boldsymbol{X}, \boldsymbol{u})\right]=\delta_{\boldsymbol{m} \boldsymbol{n}}\left(\begin{array}{c}
N \\
\boldsymbol{n}^{+}
\end{array}\right)^{-1} \prod_{i=1}^{d-1} b_{i}^{-2 n_{i}}
$$

Denote

$$
h_{\boldsymbol{n}}^{\diamond}=\left(\begin{array}{c}
N \\
\boldsymbol{n}^{+}
\end{array}\right) \prod_{i=1}^{d-1} b_{i}^{2 n_{i}} .
$$

The appropriate hypergroup property is

$$
\sum_{n} Q_{n}^{\diamond}(\boldsymbol{x}, \boldsymbol{u}) Q_{n}^{\diamond}(\boldsymbol{y}, \boldsymbol{u}) Q_{n}^{\diamond}(z, \boldsymbol{u}) h_{n}^{\diamond} \geq 0 .
$$

The first result shows that (42) is equivalent to a similar property for the original basis $\left\{u^{(l)}\right\}$. Define an orthogonal matrix as in (18) by

$$
h_{i j}=u_{j}^{(i-1)} \sqrt{p_{j}}, i, j \in[d]
$$

and set

$$
\mathfrak{s}(j, k, l)=\sum_{i=1}^{d} h_{i j} h_{i k} h_{i l} h_{i d}^{-1} .
$$


Proposition 7. For an orthonormal basis $\left\{u_{j}^{(l)}\right\}$ as in (2) with $u_{d}^{(l)} \neq 0$ for $l=0,1, \ldots, d-1$, the hypergroup property (42) holds if and only if

$$
\mathfrak{s}(j, k, l) \geq 0 \text { for all } j, k, l \in[d] .
$$

Proof A generating function proof now follows. Note that $\widehat{Q}_{\boldsymbol{n}^{+}}(\boldsymbol{x}, H)=$ $\widehat{Q}_{\boldsymbol{x}}\left(\boldsymbol{n}^{+}, H^{T}\right)$ is the coefficient of

$$
\left(\begin{array}{l}
N \\
\boldsymbol{x}
\end{array}\right) \prod_{j=1}^{d} z_{j}^{x_{j}}
$$

in

$$
\prod_{i=1}^{d}\left(\sum_{j=1}^{d} h_{i j} z_{j}\right)^{n_{i}^{+}}
$$

Multiply the sum of the triple products in (42) by

$$
\left(\begin{array}{c}
N \\
\boldsymbol{x}
\end{array}\right) \prod_{i=1}^{d} \alpha_{i}^{x_{i}}\left(\begin{array}{c}
N \\
\boldsymbol{y}
\end{array}\right) \prod_{j=1}^{d} \beta_{i}^{y_{j}}\left(\begin{array}{c}
N \\
\boldsymbol{z}
\end{array}\right) \prod_{j=1}^{d} \gamma_{i}^{z_{k}}
$$

and sum over $\boldsymbol{x}, \boldsymbol{y}, \boldsymbol{z}$ to obtain

$$
\left[\sum_{j, k, l=1}^{d} s(j, k, l) \alpha_{j} \beta_{k} \gamma_{l}\right]^{N}
$$

The coefficients of (46) in (47)) are non-negative if and only if (42) holds. The sufficiency clearly holds. For the necessity first note that for $k, j \neq d$

$$
\mathfrak{s}(d, k, k)=1, \mathfrak{s}(d, d, k)=1, \mathfrak{s}(d, k, j)=0, \mathfrak{s}(d, d, d)=1 .
$$

The coefficient of $\alpha_{j} \beta_{k} \gamma_{l}\left(\alpha_{d} \beta_{d} \gamma_{d}\right)^{N-1}$ in (47) is $N \mathfrak{s}(j, k, l)$, which is necessarily non-negative. 


\subsection{A Linearization Formula}

Linearization formulas for classical orthogonal polynomials express the product of two polynomials as a linear combination

$$
P_{i} P_{j}=\sum_{k=0}^{i+j} L_{i j k} P_{k} .
$$

Positivity and integrality of the $L_{i j k}$ is of particular interest. Background, motivation and references are in Ismail (2005) or Hounkonnou Belmedhi and Ronveaux (2000). The celebrated Littlewood-Richardson rule (Macdonald, 1998) gives a multivariate example. It expresses the product of two Schur functions. The hypergroup property allows such a result for multivariate Krawtchouk polynomials.

With notation as in section 2.3 , let $Q_{n}^{\diamond}$ be defined by (37). Set

$$
\phi_{\boldsymbol{x} \boldsymbol{y}}=m(\boldsymbol{z} ; \boldsymbol{p}) \sum_{n} Q_{n}^{\diamond}(\boldsymbol{x} ; \boldsymbol{u}) Q_{n}^{\diamond}(\boldsymbol{y} ; \boldsymbol{u}) Q_{n}^{\diamond}(\boldsymbol{z} ; \boldsymbol{u}) h_{n}^{\diamond} .
$$

From Proposition $7, \phi_{\boldsymbol{x} y}(\boldsymbol{z})$ is a probability distribution in $\boldsymbol{z}$ if and only if $\mathfrak{s}(j, k, l) \geq 0$ for all $j, k, l \in[d]$. If this is true (ie when the hypergroup property holds for the original basis $\left.\left\{u^{(l)}\right\}\right)$ then

$$
Q_{n}^{\diamond}(\boldsymbol{x}, \boldsymbol{u}) Q_{n}^{\diamond}(\boldsymbol{y}, \boldsymbol{u})=\mathbb{E}_{\phi_{\boldsymbol{x} \boldsymbol{y}}}\left[Q_{n}^{\diamond}(\boldsymbol{Z}, \boldsymbol{u})\right] .
$$

Expanding the right hand side gives a positive linearization formula.

\section{Markov Chains with Multivariate Krawtchouk Polynomial Eigen- vectors}

This section gives many natural examples of Markov chains with multivariate Krawtchouk polynomial eigenvectors. Section 3.1 reviews the work of Khare and Zhou (2009) and Zhou and Lange (2009) on composition Markov chains. These include generalized Ehrenfest urns, chains occurring in the evolution of DNA, neutral theory of biodiversity and others. These authors have used the polynomials to get sharp rates of convergence and to build martingales to calculate moments of coalescent times.

Section 3.2 develops a non-reversible theory using bi-orthogonal expansions. This is applied to generalizations of an urn model of (Mizukawa, 2010, 2011). Section 3.3 offers further generalizations all of which are diagonalized by multivariate Krawtchouk polynomials. Section 3.4 applies this construction to give a universal property of the Krawtchouk construction (and many more examples). 


\subsection{Composition Markov Chains}

Begin with a Markov chain $\boldsymbol{P}(i, j)$ with stationary distribution $\boldsymbol{p}$ on $[d]$. This induces a variety of Markov chains on the product space $[d]^{N}$. One may 'pick a coordinate at random and change that coordinate from $i$ to $j$ with probability $\boldsymbol{P}(i, j)$ '. One may change all of the coordinates in turn, independently with $\boldsymbol{P}$. More generally, one may pick a subset $S \subseteq[N]$ with probability $\mu(S)$ which is exchangeable (so $\mu(S)$ only depends on $|S|$ ) and change the values of coordinates in $S$ independently with $\boldsymbol{P}$. The symmetric group $S_{N}$ acts on $[d]^{N}$ by permuting coordinates. The orbit of a point $\boldsymbol{x} \in[d]^{N}$ is $\boldsymbol{n}=\left(n_{1}, \ldots, n_{d}\right)$ with $\boldsymbol{n}_{j}(\boldsymbol{x})=\#\left\{i: \boldsymbol{x}_{i}=j\right\}$. If $\mathbb{P}_{\mu}(\boldsymbol{x}, \boldsymbol{y})$ is the chain constructed above on $[d]$ then $\mathbb{P}_{\mu}(\boldsymbol{x}, \boldsymbol{y})=\mathbb{P}_{\mu}\left(\boldsymbol{x}^{\sigma}, \boldsymbol{y}^{\sigma}\right)$ for all $\sigma \in S_{N}$. It follows from Dynkins Criteria (Kemeny and Snell, 1976; Boyd Diaconis Parillo and Xiao, 2005) that $\mathbb{P}_{\mu}$ induces a Markov chain on the orbit space

$$
\chi(d, N)=\left\{n_{1}, \ldots, n_{d}: 0 \leq n_{i}, \sum_{i=1}^{d} n_{i}=N\right\} \text { so }|\chi(d, N)|=\left(\begin{array}{c}
d+N-1 \\
N
\end{array}\right)
$$

with a multinomial $m(\boldsymbol{x}, \boldsymbol{p})$ distribution. These are the composition chains of Khare and Zhou (2009), Zhou and Lange (2009) and Khare and Mukherjee (2013). These authors give a detailed development of many examples using the multivariate Krawtchouk polynomials to give sharp rates of convergence to stationarity and to build martingales used to bound first hitting times.

To whet the reader's appetite, here is a brief list.

Example (Ehrenfest Urns) Consider $N$ labeled balls distributed in $d$ urns. At each stage, a set of $s$ balls (say $|s|=k$ is fixed) is chosen uniformly at random and, for each ball, if in urn $i$, it is moved to urn $j$ with probability $P(i, j)$. Some special cases due to Mizukawa are considered in section 3.2.

Example (Hoare-Rahmann chain) With $N$ balls in $d$ urns, attempt to move all $N$ each time as follows; balls in urn $i$ are left fixed with probability $\alpha_{i}$ and moved with probability $1-\alpha_{i}$; if moved, they are re-distributed with probability $\left(\theta_{1}, \ldots, \theta_{d}\right)$.

Example (Evolution of DNA Chromosomes). Here a string of $N$ nucleotides labeled $\{A, T, C, G\}$ undergoes independent mutation from a fixed $4 \times 4$ transition matrix (so $d=4$ ).

Example (Lightbulb problem) There are $N$ light bulbs. At time $t$, choose a set $S_{t}$, and for $i \in S_{t}$, if the bulb is off, switch it on, if on, switch it 
off. Of interest are the total number of bulbs on at time $t$, and the first time all bulbs are off. The Krawtchouk polynomials are used to build martingales that give exact formulas for these distributions.

Example (Coalescence times for a multiperson random walk on a graph) Fix a connected simple graph $\mathcal{G}$ with $d$ vertices, distribute $N$ chips on the vertices. At each time, a randomly chosen chip picks a nearest neighbour at random and moves. Of interest is the first time all the chips are at a common vertex.

\subsection{Non-reversible Chains and Biorthogonal Expansions}

Markov chains with a Lancaster expansion are usually thought of as reversible. This section treats non-reversible chains using Biorthogonal expansions. Some examples of (Mizukawa, 2010, 2011) are treated.

Let $P=\left(P_{i j}\right)$ be a Markov transition matrix on $[d]$ with stationary distribution $\left\{p_{i}\right\}$. Suppose that $P$ is diagonalizable with left eigenvectors

$\left\{p_{i} \beta_{i}^{(k)}\right\}_{k=0}^{d-1}$, right eigenvectors $\left\{\alpha_{j}^{(k)}\right\}_{k=0}^{d-1}$ and eigenvalues $\left\{\rho_{k}\right\}_{k=0}^{d-1}$ with $\rho_{0}=$ 1. Then $P$ has spectral representation

$$
p_{i j}=p_{j}\left\{1+\sum_{k=1}^{d-1} \rho_{k} \alpha_{i}^{(k)} \beta_{j}^{(k)}\right\}, i, j=1, \ldots, d .
$$

We may also call (49) a Lancaster expansion. Examples are given below and a host of further examples of non-reversible chains with explicit real left and right eigen-vectors are in Diaconis, Pang, and Ram (2011). Of course, without reversibility, the eigen-vectors may take complex values and need not be othogonal, but they satisfy the biorthogonality relationship

$$
\sum_{i=1}^{d} p_{i} \alpha_{i}^{(k)} \beta_{i}^{(l)}=\delta_{k l}
$$

Define two sets of multivariate Krawtchouk polynomials

$$
\left\{Q_{n}(\boldsymbol{x}, \boldsymbol{\alpha})\right\},\left\{Q_{n}(\boldsymbol{x}, \boldsymbol{\beta})\right\}
$$

using the generating function (4).

Any of the schemes of section 3.1 can now be used to get a Markov chain on $\mathfrak{X}(d, N)$ with $N$ particles and a multinomial stationary distribution. These chains have spectral expansions with respect to $Q_{\boldsymbol{n}}(\boldsymbol{x}, \boldsymbol{\alpha}), Q_{\boldsymbol{n}}(\boldsymbol{x}, \boldsymbol{\beta})$. 
For example, if each of the $N$ particles independently makes a transition at each stage, the transition matrix from $\boldsymbol{x} \rightarrow \boldsymbol{y}$ is

$$
m(\boldsymbol{y} ; \boldsymbol{p})\left\{1+\sum_{\{\boldsymbol{n}: 1 \leq|\boldsymbol{n}| \leq N\}} \gamma_{\boldsymbol{n}} Q_{\boldsymbol{n}}(\boldsymbol{x}, \boldsymbol{\alpha}) Q_{\boldsymbol{n}}(\boldsymbol{x}, \boldsymbol{\beta})\right\},
$$

where

$$
\gamma_{\boldsymbol{n}}=\prod_{j=1}^{d-1} \rho_{j}^{n_{j}}
$$

Example Mizukawa (2010, 2011) considered $N$ balls distributed in $d$ urns arranged around a circle and moved to another urn in one of three schemes:

(a) a randomly chosen different urn;

(b) the next urn right $(\bmod d)$; and

(c) one of the two adjacent urns $(\bmod d)$ with equal probability.

All of these examples have transition matrices for a single ball change which are circulants (Davis, 1979). Let $\boldsymbol{P}$ be a general $d \times d$ circulant transition matrix with first row $\left\{q_{j}\right\}_{j=1}^{d}$ and other rows rotated successively from the first, so the $i^{\text {th }}$ row is $\left\{q_{j-i}\right\}_{j=1}^{d}$ with subscripts taken mod $d$. $\boldsymbol{P}$ is doubly stochastic, with a uniform stationary distribution on $[d]$. An eigenfunction expansion of $\boldsymbol{P}$ is

$$
p_{a b}=\frac{1}{d} \sum_{k=0}^{d-1} \eta_{k} e^{2 \pi i k(a-1) / d} e^{-2 \pi i k(b-1) / d}, a, b=1, \ldots, d,
$$

where

$$
\eta_{k}=\sum_{r=0}^{d-1} q_{r+1} e^{2 \pi i r k / d} .
$$

Here $\boldsymbol{P}$ is reversible if and only if it is symmetric. (eg cases (a), (c)).

Construct the multivariate Krawtchouk polynomials (51) by taking

$$
\alpha_{a}^{(l)}=e^{2 \pi i l(a-1) / d}, \beta_{b}^{(l)}=e^{-2 \pi i l(b-1) / d}, l=0, \ldots, d-1, a, b \in[d] .
$$

Then the representation (52) holds with

$$
\gamma_{\boldsymbol{n}}=\sum_{l=0}^{d-1} \eta_{l} \frac{n_{l}}{N}
$$


In this circulant case, the eigenfunctions $Q_{\boldsymbol{n}}$ are the monomial symmetric functions as recognized by Mizukawa. To see this, label the balls so that $Z_{l}=k$ if ball $j$ is in urn $k$. Then from (24)

$$
Q_{\boldsymbol{n}}(\boldsymbol{X}, \boldsymbol{u})=\sum_{\left\{A_{l}\right\}} \prod_{k_{1} \in A_{1}} e^{(2 \pi i / d)\left(Z_{k_{1}}-1\right)} \cdots \prod_{k_{d-1} \in A_{d-1}} e^{(2 \pi i(d-1) / d)\left(Z_{k_{d-1}}-1\right)}
$$

where the summation is over subsets of $[n],\left\{A_{l}\right\}$ with $\left|A_{l}\right|=n_{l}, 1 \leq$ $l \leq d$. Let $n_{0}=N-|\boldsymbol{n}|$, so $\left\{n_{j}\right\}_{j=0}^{d-1}$ is a composition of $N$. Regard $\left(Z_{k}-1\right)_{k=1}^{N}$ as a partition $0^{x_{1}} 1^{x_{2}} \cdots(d-1)^{x_{d}}$. Let $\xi=e^{2 \pi i / d}$ and $\Xi=$ $\left((1)^{n_{0}}(\xi)^{n_{1}} \cdots\left(\xi^{d-1}\right)^{n_{d-1}}\right)$ then

$$
Q_{n}(\boldsymbol{X}, \boldsymbol{\alpha})=m_{n}(\Xi)
$$

It is plausible that very similar results hold if $P$ is a $\mathcal{G}$-circulant Diaconis, 1990).

\subsection{Further Generalizations}

There is a natural generalization of the processes in sections 3.1, 3.2 that leads to Markov chains with Krawtchouk eigen-functions. Fix a basis $\left\{u^{(l)}\right\}$, orthonormal with respect to $\boldsymbol{p}=\left(p_{1}, \ldots, p_{d}\right)$. Let $\mathcal{L}(\boldsymbol{\beta}, \boldsymbol{u})=\left\{\left(\beta_{1}, \ldots, \beta_{d-1}\right)\right\}$ such that

$$
K_{\boldsymbol{\beta}}(i, j)=p_{j}\left\{1+\sum_{l=1}^{d-1} \beta_{l} u_{i}^{(l)} u_{j}^{(l)}\right\}=0 .
$$

This $\mathcal{L}$ is a non-empty, compact, convex set, the Lancaster set for $\left\{u^{(l)}, \boldsymbol{p}\right\}$ (Koudou, 1996). Observe that $\boldsymbol{\beta} \in \mathcal{L}$ implies that $K_{\boldsymbol{\beta}}$ is a reversible Markov Kernel with $\boldsymbol{p}$ a stationary distribution and $\left\{u^{(l)}\right\}$ as (right) eigen-functions. Since $\boldsymbol{\beta}$ are the eigen-values, $-1 \leq \beta_{i} \leq 1$. $\mathcal{L}$ contains 1 in an open neighbourhood of zero and if $\boldsymbol{\beta}=(1, \ldots, 1), K_{\boldsymbol{\beta}}$ is allowable. Finally, $\mathcal{L}$ is closed under coordinate-wise product (Hadamard product) and so forms a commutative semi-group with 1 as identity. Further $K_{\boldsymbol{\beta}} K_{\boldsymbol{\gamma}}=K_{\boldsymbol{\gamma}} K_{\boldsymbol{\beta}}=K_{\boldsymbol{\beta} \circ \boldsymbol{\gamma}}$. Determining an exact description of $\mathcal{L}$ is an ongoing research area. See Bakrv and Huet (2008) and Ismail (2005) section 4.7 and the references in Diaconis, Khare and Saloff-Coste (2008). If the $\left\{u^{(l)}\right\}$ satisfy the hypergroup property

$$
\sum_{l=0}^{d} \frac{u_{i}^{(l)} u_{j}^{(l)} u_{k}^{(l)}}{u_{i_{0}}^{(l)}} \geq 0
$$


for some fixed $i_{0}$ and all $i, j, k \in[d]$, then the extreme points of $\mathcal{L}$ are

$$
\boldsymbol{\beta}=\left(\frac{u_{1}^{(l)}}{u_{i_{0}}^{(l)}}, \ldots, \frac{u_{d}^{(l)}}{u_{i_{0}}^{(l)}}\right), 0 \leq l \leq d-1 .
$$

See Bakry and Huet (2008) for a proof and section 2.2 for examples.

With $\mathcal{L}$ in focus, a general class of Markov chains with multinomial $(N, \boldsymbol{p})$ stationary distributions and Krawtchouk polynomial eigen-functions can be defined.

Let $\mu$ be an exchangeable probability on $\mathcal{L}^{N}$. Define $K_{\mu}(\boldsymbol{x}, \boldsymbol{y})$ on $\mathfrak{X}(N, d)$ as the orbit chain on $[d]^{N}$ derived from picking $\left(\boldsymbol{\beta}_{1}, \ldots, \boldsymbol{\beta}_{d}\right)$ from $\mu$ and moving the $j^{\text {th }}$ coordinate with $K_{\boldsymbol{\beta}_{j}}\left(j, j^{\prime}\right)$. The eigen-values of the product chain are

$$
\lambda_{i_{1} \ldots i_{N}}=\int \boldsymbol{\beta}_{i_{1}} \cdots \boldsymbol{\beta}_{i_{d}} \mu\left(d \boldsymbol{\beta}_{1} \cdots d \boldsymbol{\beta}_{N}\right) .
$$

Because of exchangeability, this only depends on $\boldsymbol{i}=\left(i_{1}, i_{2}, \ldots, i_{N}\right)$ through $n_{1}(\boldsymbol{i}), \ldots, n_{d}(\boldsymbol{i})$. This defines

$$
\lambda_{n_{1} \ldots n_{d}}
$$

Summarizing:

Proposition 8. Let $\left\{u^{(l)}\right\}_{l=0}^{d-1}$ be an orthonormal set on $[d]$ with repect to $\boldsymbol{p}$. Let $\mu$ be an exchangeable probability on $\mathcal{L}^{N}$. The Markov chain $K_{\mu}$ on $\mathfrak{X}(N, d)$ has a $m(\boldsymbol{x}, \boldsymbol{p})$ stationary distribution, multinomial Krawtchouk eigen-functions $\left\{Q_{\boldsymbol{n}}(\boldsymbol{x}, \boldsymbol{u})\right\}_{|\boldsymbol{n}| \leq N}$ with eigenvalues $\left\{\lambda_{\boldsymbol{n}}\right\}_{|\boldsymbol{n}| \leq N}$.

This construction includes all of the examples in section 3.1. It shows that the multivariate Krawtchouk polynomials have a kind of universal quality, diagonalizing the orbit chains of arbitrary products of Markov chains with $\left\{u^{(l)}\right\}$ as eigen-vectors.

The set of all exchangeable probabilities $\mu$ on $\mathcal{L}^{N}$ is a convex simplex whose extreme points are straightforward to describe: put $N$ balls in an urn labeled with $\boldsymbol{\beta}^{1}, \boldsymbol{\beta}^{2}, \ldots, \boldsymbol{\beta}^{N}$ with $\boldsymbol{\beta}^{i} \in \mathcal{L}$ and draw them out sampling without replacement (Diaconis and Freeman, 1980).

\section{Acknowledgment}

Thanks to Yuan Xu for his substantial comments on the paper and to Pratha Dharmawansa for his carefull reading and list of corrections. Robert Griffiths was supported by the Department of Statistics, Stanford University in 2011; the Miller Foundation, Berkeley, in a visit to the Department of Statistics, 
Berkeley in 2012; the Clay Mathematics Institute in a visit to the University of Montreal in 2013; and the Institute of Statistical Mathematics, Tokyo, in 2014. He thanks the institutions for their support and hospitality.

\section{References}

Aitken, A. C. And Gonin, H. T. (1935) On fourfold sampling with and without replacement. Proc. Roy. Soc. Edinb. 55 114-125.

Askey, R. (1975) Orthogonal polynomials and special functions. SIAM

Bakry, D. And Huet, N. (2006) The hypergroup property and representation of Markov Kernels. Séminare de Probabilitiés XLI, Lecture notes in Mathematics, Vol 1934, 295-347, Springer.

Bochner, S. (1954) Positive zonal functions on spheres. Proc. Nat. Acad. Sci. USA 40 1141-1147.

Boyd, S., Diaconis, P., Parillo, P. And Xiao, L. (2005) Symmetry analysis of reversible Markov chains. J. Internet math.

Chinara, T. (1978) An introduction to orthogonal polynomials. Gordon and Breach, New York.

Davis, P (1979) Circulant matrices. Wiley, New York.

Desai, R. (2010) Quantum mechanics with basic field theory. Cambridge.

Diaconis, P. (1988) Group representations in probability and statistics. Institute of Mathematical Statistics Lecture Notes-Monograph Series, 11. Institute of Mathematical Statistics, Hayward, CA.

Diaconis, P. (1990) Patterned matrices. Proc. Symp. Appl. Math. 40 3758.

Diaconis, P. And Freedman, D. (1980) Finite exchangeable sequences. Ann. Probab. 8 745-764.

Diaconis, P. And Griffiths R. C. (2012) Exchangeable pairs of Bernoulli random variables, Krawtchouk polynomials, and Ehrenfest urns. Aust. NZ J. Stat. 54 81-101.

Diaconis, P., Khare, K. And Saloff-Coste, L. (2008) Gibbs sampling, exponential families and orthogonal polynomials. Statist. Sci., 23 151200. 
Diaconis, P., Pang, C. A., And Ram, A. (2011). Hopf algebras and Markov chains: two examples and a theory. Journal of Algebraic Combinatorics $1-59$.

DunkL, C. F., AND RAmirez, D. E. (1974). Krawtchouk polynomials and the symmetrization of hypergroups. SIAM Journal on Mathematical Analysis, 5 351-366.

DunkL, C. AND Xu, Y. (2001) Orthogonal polynomials of several variables. Cambridge University Press.

Eagleson, G. K. (1964) Polynomial expansions of bivariate distributions. Ann. Math. Statist. 35 1208-1215.

Eagleson, G. K. (1969) A characterization theorem for positive definite sequences on the Krawtchouk polynomials. Austral. J. Statist. 11 29-38.

Feynman, R. P. (1972). Statistical Mechanics, A Set of Lectures, California, Institute of Technology.

Genest, V., Vinet, L. And Zhedanov, A. (2013). The multivariate Krawtchouk polynomials as matrix elements of the rotation group representations on oscillator states. arXiv:1306.4256.

Griffiths, R. (1971) Orthogonal polynomials on the multinomial distribution. Austral. J. Statist. 13 27-35. Corrigenda (1972) Austral. J. Statist. 14270 .

GRIFFITHS, R. (1975). Orthogonal polynomials on the negative multinomial distribution. J. Multivariate Anal. 5 271-277.

Grunbaum, F. And Rahman, M. (2011) A system of multivariable Krawtchouk polynomials and a probabilistic application. SIGMA 7 119136.

Hoare, M. And Rahman, M. (1983) Cumulative Bernoulli trials and Krawtchouk processes. Stochastic Process. Appl. 16 113-139.

Hoare, M., Rahman, M. (2008). A probabilistic origin for a new class of bivariate polynomials. SIGMA 418 pages.

Hounkonnou, M., Belmedhi, S. And Ronveaux, R. (2000) Linearization of arbitrary products of classical orthogonal polynomials. Applicationes Mathematicae, 27 187-196. 
IsAacs, I. (1994). Character Theory of Finite Groups. Dover. New York.

Ismail, M. E. H. (2005) Classical and Quantum Orthogonal Polynomials in one variable, Volume 98 of Encyclopedia of Mathematics and its Applications. Cambridge University Press.

James, G., And Liebeck, M. (2001). Representations and characters of groups. Cambridge University Press.

Kemeny, J. And Snell, L. (1976) Finite Markov chains. New York: Springer-Verlag.

Khare, K. And MukherJee (2013) Convergence analysis of some multivariate Markov chains using stochastic monotonicity. Ann. Appl. Probab. 23 811-833.

Khare, K. And Zhou, H. (2009) Rates of convergence of some multivariate Markov chains with polynomial eigenfucntions. Ann. Appl. Probab. 19 $737-777$.

Koekoek, R., Lesky, P. And Swanttouw, N. (2010) Hypergeometric orthogonal polynomials and their $q$-analogues. Springer, Berlin.

Koudou, A. (1996) Probabilitiés de Lancaster, Exposition. Math. $1247-$ 275.

Lancaster, H. (1965). The Helmert matrices. Amer Math. Monthly 72, $4-12$.

Lancaster H. (1969) The chi-squared distribution, John Wiley \& Sons.

LiU, J. (1996) Metropolized independent sampling with comparisons to rejection sampling and importance sampling. Statistical. Comput. 6 113119.

Macdonald, I. (1998) Symmetric functions and Hall polynomials, 2nd ed. Oxford University Press.

Mizukawa, H. (2010) Finite Gelfand pair approaches for Ehrenfest diffusion model. arXiv:1009.1205.

MizukawA, H. (2011) Orthogonality relations for multivariate Krawtchouk polynomials. arXiv:1009.1203. 
Mizukawa, H. And TANakA, H. (2004). $(n+1, m+1)$ - hypergeometric functions associated to character algebras. Proceedings of the American Mathematical Society 132, 2613-2618.

Reed, M. And Simon, B. (1975) Methods of Modern Mathematical Physics: Vol. 2.: Fourier Analysis, Self-Adjointness. Academic Press.

Saltzman, J. (2010) PhD Thesis, Department of Statistics, Stanford University.

Stein, C. (1992). A way of using auxiliary randomization. Probability Theory (Singapore, 1989), 159-180.

Vere-Jones, D. (1971) Finite bivariate distributions and semigroups of non-negative matrices. Q. J. Math. 22 247-270.

XU, Y. (2013) Hahn, Jacobi, and Krawtchouk polynomials of several variables. arXiv:1309.1510v1

Zhou, H. And Lange, K. (2009) Composition Markov chains of multinomial type. Adv. Appl. Probab. 41 270-291. 